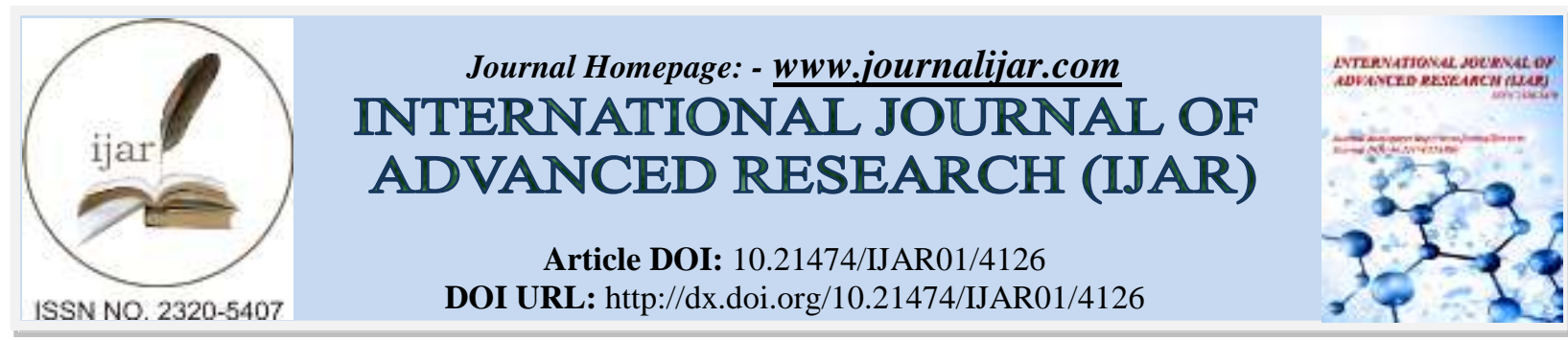

RESEARCH ARTICLE

\title{
CURRENT PHYSIOTHERAPY MANAGEMENT OF INFANTS WITH TORTICOLLIS AMONG PHYSIOTHERAPY PRACTITIONERS IN SRI LANKA.
}

Dissanayake DMRP ${ }^{1}$, Fontaine ME ${ }^{1}$, Madushani AG ${ }^{1}$, Nishshanka NACL ${ }^{1}$, Perera ADP ${ }^{1}$ and Rodrigo $I^{2}$.

1. Department of Physiotherapy, Faculty of Allied Health Sciences, General Sir John Kotelawla Defence University, Sri Lanka.

2. Faculty of Medicine, General Sir John Kotelawla Defence University, Sri Lanka.

\section{Manuscript Info}

Manuscript History

Received: 07 March 2017

Final Accepted: 04 April 2017

Published: May 2017

Key words:-

Congenital muscular torticollis,

Physiotherapy management \& treatment,

Assessment tools

\begin{abstract}
Torticollis is the third most frequently occurring musculoskeletal condition caused by unilateral shortening of the sternocledomastoid muscle. The aim of this study is to identify different physiotherapy assessment tools used in Sri Lanka for torticollis, to identify barriers / issues for management and determination of current physiotherapy management for Congenital Muscular Torticollis (CMT). The descriptive cross sectional study was conducted with 227 registered physiotherapy practitioners who are handling torticollis patients. The self-administered questionnaire was used to gain information of assessment tools, treatment techniques and issues faced when managing patients with CMT. The most respondents used visual estimate as the assessment tool for the torticollis patients $(98.2 \%)$. All the respondents reported that they were using combination of multiple treatment techniques when treating infants with torticollis as handling techniques (98.2\%), neck passive range of motion exercises (97.8\%), positioning (97.3\%), sternoclidomastoid muscle massage (96.9\%), manual stretching (95.6\%), and environmental adaptation (93.4\%). Poor home management was found as the major issue faced by the physiotherapist when treating CMT (81.9\%). The majority of physiotherapists used visual estimate as the assessment tool to assess CMT patients. It is recommended to practice to use of multiple treatment interventions. The Lack in proper cervical ROM assessment was identified and international protocols can be used to increase measurement accuracy. Poor home management, observed as the main issue faced by physiotherapists and may be corrected by the use of appropriate handouts for parents.
\end{abstract}

Copy Right, IJAR, 2017,. All rights reserved.

\section{Introduction:-}

Torticollis is a comparatively common neck condition of infancy that is caused by idiopathic, unilateral shortening of the sternoclidomastoid muscle. This condition is detected at birth or shortly after birth. Infant will generally present with persistent asymmetrical head posture of lateral flexion to the affected side and rotation to the opposite side (Cheng et al,2000). Torticollis is the third most frequently occurring musculoskeletal condition in infants with a

Corresponding Author:- Perera ADP.

Address:- Department of Physiotherapy, Faculty of Allied Health Sciences, General Sir John Kotelawla 419 
reported incidence of $0.4 \%$ to $1.9 \%$ (Burch et al.,2009). The incidence of CMT ranges from 0.3 to $2 \%$ of newborns, but has been reported as high as $16 \%$ (Do TT,2006).

In many cases, a mass or tumor can be palpated in the involved muscle. There are two types of torticollis as congenital muscular torticollis and acquired torticollis. The type and the presentation of the age give the highly predictive of the time required to resolve ROM limitations. Early diagnosis will helpful to resolve the condition within short time period of treatments. The evidence is strong that earlier intervention results in the best outcomes (Petronic et al., 2010). The exact etiology of this condition remains unknown so far more than 80 entities have been described that can cause torticollis (Petronic et al., 2010).

Most cases of torticollis respond very well to physical therapy interventions in world wide. Because conservative management has been so successful, surgery is typically done only in severe cases (Emery et al,1994). In Examination, physical therapist use goniometer, arthrodial protracter or tape to take range of motion measurements. Those data were used to plan correct physiotherapy treatment program for the child. Each patient with CMT has undergone assessment individually as the treatments may vary patient to patient with age, etiology and with other factors.

According to our knowledge there were no studies found on current physiotherapy management for torticollis in Sri Lanka. Therefore; it is important to determine the current physiotherapy management of infants with torticollis among physiotherapists in Sri Lanka, to identify different assessment tools used by physiotherapists, different treatment techniques for the management, and the barriers / issues for management of torticollis.

\section{Material and Method:-}

The descriptive cross sectional study was conducted with 300 registered government physiotherapists who are handling torticollis patients in Sri Lanka. The self-administered questionnaire was designed with current available literature and with the guidance of clinical expert in the physiotherapy field. The pilot study was conducted to ensure content validity of the questionnaire and develop or modify questions. The questionnaire was used to gain information of assessment tools, treatment techniques and issues faced when managing patients with CMT. Ethical clearance was obtained from ethical review committee, Faculty of Medicine of General Sir John Kothelawala Defence University. The data was entered and analyzed in SPSS (Statistical Package for Social Sciences (SPSS) version 17.0 database. Descriptive statistics were used to describe and summarize the assessment tools, treatment techniques and issues faced when managing patients with CMT.

\section{Results:-}

300 questionnaires were sent to the physiotherapists who are handling torticollis patients and 227 participants responded $(75.6 \%)$. The distribution of physiotherapists who treat torticollis according to the type of hospital was assessed. The results show $54.2 \%$ in teaching hospital, similar percentages of $21.2 \%$ in general and based hospitals and minimal number of physiotherapists $(3.5 \%)$ in other hospitals.

Number of patients were treated by a physiotherapist per week was assessed. $67.8 \%$ of physiotherapists have treated torticollis less than 3 patients per week while $21.6 \%$ of physiotherapists have treated more than 3 patients per week and $9.7 \%$ of physiotherapists treated more than 6 torticollis patients per week. Frequency of visits to the physiotherapy department for the treatments was assessed. $50.7 \%$ of patients visited 3 times per week and $0.4 \%$ of patients visit 3 times per week. In $18.5 \%$ of patients' treatment sessions per week depends according to the severity of the condition.

Assessment tools were used by the physiotherapists when assessing patients with CMT was assessed. Comparing to other tools; $98.2 \%$ of Sri Lankan physiotherapists used visual estimate to assess torticollis patients. Measuring tape was used by $43.6 \%$ of physiotherapists and $34.4 \%$ of physiotherapists used video records and photography. The minimal percentage $(0.9 \%)$ obtained for athrodialprotactorto assess torticolis by palpation.

Different treatments methods used by the physiotherapists when managing patients with CMT was assessed. Handling techniques was the major treatment technique used by the physiotherapists $98.2 \%$. There were other popular treatment methods as neck passive range of motion exercises (97.8\%), positioning (97.4\%),sternoclidomastoidmuscle massage (96.9\%), manual stretching (95.6\%), and environmental adaptation 
(93.4\%). Microcurrent treatment was the lowest modality used by thephysiotherapists (0.9\%) to treat torticolis.Issues faced by the physiotherapists when managing patients with CMT was assessed. Poor home management had highest percentage $81.9 \%$ compared to the other issues.Lack ofparent education $(79.7 \%)$ and delay in diagnosing $(63.4 \%)$ also can be observed as main issues.

\section{Discussion:-}

Assessment of patient is the most important part when treating CMT patients. As they are pediatric patients it will be more challengeable. Except subjective and objective assessment in examination cervical mobility should be measured. Freed, and Coulter, (2004) mentioned that measuring the range of motion is a main part of CMT assessment.

The present study found that almost all respondents assessed CMT by visual estimate (98.2\%) while the other commonly used tools are measuring tape (43.6\%) photographic and video graphic records (34.4\%). Comparatively, very less amount of physiotherapists using goniometer (5.3\%) or arthrodial protractor(0.9\%). Luxford et al., (2009) found that most of practitioners in New Zealand used visual estimate (86\%) as a measurement tool due to absence of suitable measurement tool specially for the infants and 95\% participants never used arthrodial protractor and $53 \%$ never used goniometer as an assessment tool. The reliability of visual estimate in cervical ROM in CMT has not been established. Limited research into the use of arthrodial protractor and goniometer has been undertaken. Klackenberg et al (2005) reported that the measurement of cervical ROM in CMT and found that the arthrodial protractor and goniometer had high intra-rater reliability. The measurement protocols used by Klackenberg et al., (2005) are similar to those described by Cheng et al (2000) and Emery, (1994). The clinical practice guidelines for physical therapy management of torticollis developed by the American Physiotherapy Association stated that measurement of passive ROM using the arthrodial protractor has recently been recommended.

The present study stated that physiotherapists used multiple treatment inventions to treat torticollis. The commonest treatments techniques used were handling techniques $(98.2 \%)$; passive range of motion exercises to neck (97.8\%), positioning (97.4\%), sternocleidomastoid muscle massage (96.9\%), manual stretching (95.6\%) and advising on home environmental modifications (95.2\%) and handling advices (95.2\%). Only four (1.8\%) physiotherapists used handouts to educate parents and other less frequently used treatment technique was tubular orthosis (4\%). Luxford et al., 2009 frequently used treatments technique were handling advices (95\%), providing handouts (86\%), positioning (76\%), facilitate cervical strengthening exercises $(67 \%)$ and midline activities $(67 \%)$. But, this was not consisting with the present study found that providing handouts were $1.8 \%$. It is found that understanding and compliance was increased when parents were provided with adequate information and instructions (Nader, 1985). Clinical Practice Guidelines for Physical Therapy Management of Torticollis stated the treatment primarily focused on normalization of active and passive cervical ROM and muscle function. Parental education, handling, and strength and stretching exercises were key components of the treatment protocol. Kim et al. (2009) concluded that micro current therapy is more effective in improving therapeutic compliance in infants with torticolis than traditional therapy. In Sri Lanka micro current therapy is used as a treatment modality for torticollis only $0.9 \%$ while ultrasound therapy for only $33.9 \%$. Traction using body weight could be identified as a new technique used by the physiotherapists (15.4\%) in Sri Lanka, but in the literature there were no any article or study on this technique. Further studies should be conducted to validate this treatment method.

Muscle function scale (MFS) was used to assess the degree of symmetry between muscle function of the lateral flexors of the affected compared with the unaffected side (Kaplan et al, 2013). In Sri Lanka there was no scale as MFS to assess CMT patients according to our knowledge.

According to the present study, key issues identified to treat CMT patients was the poor home management $81.9 \%$. The second issue was lack of parental education. Petronic et al., (2010) also found that the parental compliant is mandatory for successful outcome while Luxford et al.,(2009) stated that lack of parent compliance was a main issue during practice $(43 \%)$ and limited level of parent understanding $(38 \%)$ as the other issue. There may be interrelationship between poor home management and parent's education. Further studies should be conducted on effectiveness of parent's education and its effect on home management. Other issues faced by Sri Lankan Physiotherapist were delay in diagnosis (63.4\%) and lack of time (41.4\%). 


\section{Conclusion:-}

Majority of the physiotherapists treated less than 3 patients with CMT per week and most of the CMT patients visited physiotherapy department 2 times per week for the treatments. Visual estimate was the commonest assessment tool among physiotherapist in Sri Lanka to assess CMT patients. The Lack in proper cervical ROM assessment was identified and international protocols can be used to increase measurement accuracy. It is recommended to practice multiple treatment interventions as handling techniques, neck passive range of motion exercises, positioning, sternoclidomastoid muscle massage, manual stretching, and environmental adaptation in Sri Lanka. Poor home management was observed as the main issue faced by physiotherapists and it is suggested to use of appropriate handouts to educate parents.

\section{Acknowledgement:-}

The authors would like to acknowledge all the participants for their support and collaboration during the study.

\section{References:-}

1. Burch C., Hudson, P., Reder, R., Ritchey, M., \&Strenk, M(2009): Therapy management of congenital muscular torticollis. Cincinnati children's hospital medical center: evidence based clinical care guideline for therapy management of congenital muscular torticollis.33 pp 113.

2. Cheng, J.C., Tang, S.P., Chen, T.M., Wong, M.W., \& Wong, E.M(2000): The clinical presentation and outcome of treatment of congenital muscular torticollis in infants- a study of 1,086 cases. 35(7) pp 1091-1096.

3. Do, T.T(2006): Congenital muscular torticollis: current concepts and review of treatment 18(1) pp 26-29.

4. Emery, C (1994): The determinants of treatment duration for congenital muscular torticollis. 74 (10), pp 921929.

5. Freed, S.S., \& Coulter, C(2004): Identification and treatment of congenital muscular torticollis in infants, Orthotics and prosthetics department, Children's healthcare of Atlanta. 16(4), pp 18-23.

6. Kaplan, S.L., Coulter, C., \& Fetters, L(2013): Physical therapy management of congenital muscular torticollis: An evidence-based clinical practice guideline. 25(4), pp 348-394.

7. Kim, M.Y., Kwon, D.R., \& Lee H(2009): Therapeutic effect of microcurrent therapy in infants with congenital muscular torticollis. 1(8), pp 736-739.

8. Klackenberg, E.P., Elfving, B., Haglund, A.Y., \&Carlberg, E.B(2005): Intra-rater reliability in measuring range of motion in infants with congenital muscular torticollis.7 pp 84-89.

9. Luxford, B.K., Hale, L., \&Piggot, J(2009): The physiotherapy management of infants with congenital muscular torticollis: a survey of current practice in New Zealand. 37(3), pp 127- 135.

10. Nader,P.R,(1985):Improving the practice of pediatric patient education:a synthesis and selective review.Preventive Medicine 14,pp 688-701

11. Petronic, I., Brdar, R., Cirovic, D., Lukac, M., Janic, D., Pavicevic, P., Golibovic, Z., \&Knezevic, T(2010): Congenital muscular torticollis in children: distribution, treatment duration and outcome. 46(2) pp 153-157. 